\title{
Gaze-independent ERP-BCls: augmenting performance through location-congruent bimodal stimuli
}

\author{
Marieke E. Thurlings ${ }^{1,2}$ *, Anne-Marie Brouwer' ${ }^{2}$, Jan B. F. Van Erp ${ }^{2}$ and Peter Werkhoven ${ }^{1}$ \\ 1 Information and Computing Sciences, Utrecht University, Utrecht, Netherlands \\ ${ }^{2}$ Perceptual and Cognitive Systems, TNO, Soesterberg, Netherlands
}

\author{
Edited by: \\ Mikhail Lebedev, Duke University, \\ USA

\section{Reviewed by:} \\ Dewen Hu, National University of \\ Defense Technology, China \\ Betts Peters, Oregon Health and \\ Science University, USA \\ *Correspondence: \\ Marieke E. Thurlings, Information \\ and Computing Sciences, Utrecht \\ University, P. O. Box 80.089, 3508 \\ TB Utrecht, Netherlands \\ e-mail:marieke.thurlings@ \\ gmail.com
}

\begin{abstract}
Gaze-independent event-related potential (ERP) based brain-computer interfaces (BCls) yield relatively low $\mathrm{BCl}$ performance and traditionally employ unimodal stimuli. Bimodal ERP-BCls may increase $\mathrm{BCl}$ performance due to multisensory integration or summation in the brain. An additional advantage of bimodal $\mathrm{BCls}$ may be that the user can choose which modality or modalities to attend to. We studied bimodal, visual-tactile, gaze-independent $\mathrm{BCls}$ and investigated whether or not ERP components' tAUCs and subsequent classification accuracies are increased for (1) bimodal vs. unimodal stimuli; (2) location-congruent vs. location-incongruent bimodal stimuli; and (3) attending to both modalities vs. to either one modality. We observed an enhanced bimodal (compared to unimodal) P300 tAUC, which appeared to be positively affected by location-congruency $(p=0.056)$ and resulted in higher classification accuracies. Attending either to one or to both modalities of the bimodal location-congruent stimuli resulted in differences between ERP components, but not in classification performance. We conclude that location-congruent bimodal stimuli improve ERP-BCls, and offer the user the possibility to switch the attended modality without losing performance.
\end{abstract}

Keywords: BCl, ERP, gaze-independent, bimodal, tactile, multisensory, location-congruency, selective attention

\section{INTRODUCTION}

Event-related potential (ERP) based brain-computer interfaces (BCIs) can be used to actively and voluntarily control a system, e.g., for communication (Farwell and Donchin, 1988) or navigation (Bell et al., 2008; Thurlings et al., 2010). ERP-BCIs make use of stimuli that correspond to control options (e.g., "left" or "right"). The user can select an option by attending to the corresponding stimulus (target) while ignoring other stimuli (nontargets). Stimulus-locked brain responses (ERPs) differ between the attended targets and ignored nontargets.

Most ERP-BCIs employ visual stimuli and require the user to gaze at the target stimulus, i.e., such a BCI is gaze-dependent. When the user does not directly gaze at the target but only covertly attends to it, the high-level endogenous ERP components but not the low-level perceptual ERP-components differ from those of nontargets. This results in a reduced BCI performance in terms of classification accuracy (and hence bitrate) (Brunner et al., 2010; Treder and Blankertz, 2010). BCIs for which users do not have to gaze at the stimuli or to shift focus (alter viewing direction) in order to control it are called gaze-independent. An example is the Hex-o-Spell of Treder and Blankertz (2010). The importance of developing BCIs independent of the ability to shift focus has been expressed in studies investigating the rapid serial visual presentation paradigm (Orhan et al., 2012; Acqualagna and Blankertz, 2013). Yet, in that paradigm participants are required to directly focus at the visual stimuli.
When users cannot reliably direct their gaze, or when other tasks interfere with gaze, stimuli can also be presented in other modalities like the auditory modality (Schreuder et al., 2010, 2011; Höhne et al., 2011). In application domains such as driving and gaming, BCIs must be gaze-independent as gaze is required for control and navigation tasks and the visual (and auditory) channel is already heavily loaded (Van Erp and Van Veen, 2004). The tactile channel has also been suggested as a viable alternative for these situations, and Brouwer and Van Erp (2010) demonstrated the feasibility of employing tactile stimuli around the waist in a tactile ERP-BCI. The natural correspondence of tactile stimuli around the waist with navigation directions (Van Erp, 2005) makes a tactile ERP-BCI especially interesting for navigation applications.

BCI performance of tactile ERP-BCIs (Brouwer et al., 2010; Thurlings et al., 2012a,b) is generally lower than that of gazedependent BCIs (Thurlings et al., 2012a,b). In addition, when a $\mathrm{BCI}$ is used as a control device in the context of a dual-task, for example to navigate in a game, BCI performance is even lower than in BCI-only tasks (Thurlings et al., 2013). Therefore, in order to achieve effective use of BCI outside the lab, it is highly important to increase BCI performance of gaze-independent BCIs.

This study focusses on potential benefits regarding brain activity resulting from stimulus presentation in multiple sensory modalities, using a gaze-independent setup, and addresses 
three main research questions which are introduced in the next sections.

\section{THE BIMODAL ERP-BCI}

The processing and integration of multisensory stimuli is likely to cause additional neuronal activity (Ernst and Bülthoff, 2004; Driver and Noesselt, 2008; Stein and Stanford, 2008). Integration may take place at perceptual stages (Molholm et al., 2002; Philippi et al., 2008), higher cognitive stages (Schröger and Widmann, 1998), and/or during motor preparation and execution (Giray and Ulrich, 1993). Bimodal stimuli generally yield faster behavioral responses and more accurate perceptual discrimination (Gondan et al., 2005; Teder-Sälejärvi et al., 2005; Philippi et al., 2008).

Multisensory integration has extensively been investigated in cognitive science, but has barely received attention in the field of BCI. In a recent BCI-study, we showed that an additional (early) ERP component was present when participants were presented with and attended to stimuli in two modalities rather than one, due to multisensory interaction (Thurlings et al., 2012a). To the best of our knowledge, only two other BCIrelated studies investigated bimodal stimuli: visual-tactile stimuli (Brouwer et al., 2010), and audio-visual stimuli (Belitski et al., 2011). In both studies the authors reported increased classification accuracies (i.e., the percentage of correctly classified target responses) for bimodal compared to unimodal conditions, which is in line with the trend we reported in Thurlings et al. (2012a).

Multisensory or bimodal ERP-BCIs can be regarded as a type of hybrid BCIs (Pfurtscheller et al., 2010a). Hybrid BCIs are BCIs that "can either process their inputs simultaneously, or operate two systems sequentially". By allowing the processing of two inputs simultaneously, the second input could improve the classification accuracy of the first BCI (Pfurtscheller et al., 2010a; Yin et al., 2013).

As motivated in the beginning of the introduction, we are interested in multisensory BCIs, as a way to potentially increase BCI performance of (traditional) unimodal ERP-BCIs, in particularly the gaze-independent variants. From the three above mentioned bimodal studies, only Brouwer and Van Erp (2010) used a gaze-independent setup with visual-tactile stimuli. However in that study the effects on target and non-target responses of endogenous (voluntary) attention and exogenous (stimulus driven) attention were confound. Both endogenous and exogenous attention can affect ERP components (Woldorff and Hillyard, 1991), but only endogenous attention is relevant for BCI operation. Thus, the question whether or not gaze-independent ERP-BCIs benefit from bimodal stimulus presentation remains unanswered.

Multisensory integration has been shown to start as early as 80-120 ms after stimulus onset for visual-tactile stimuli (Sambo and Forster, 2009), but is modulated by endogenous attention at different stages of processing (Talsma and Woldorff, 2005). As reported in Thurlings et al. (2012a), positive effects of bimodal stimulus attending have been shown on an early stage of processing, i.e., early negative activity (N1) in the difference ERP (target minus nontarget ERP) was stronger for the bimodal compared to the unimodal conditions. However, we observed negative effects of bimodal stimulus attending on a late stage of the ERP, i.e., positive late activity (P300) in the difference ERP was stronger for one of the unimodal conditions (visual) compared to the bimodal condition. We hypothesized that the latter effect was caused by the spatial relation of the two unimodal stimuli that formed a bimodal stimulus pair. More specifically, although the spatial relation unambiguously indicated which unimodal stimuli formed a pair, those stimuli were not co-located. Possibly, this affected spatial attention and top-down stimulus processing. Therefore in the present study we focus on co-located (i.e., location-congruent) bimodal stimuli, with the expectation to lose the negative effects on late ERP components.

Thus, our first research question is: Are ERP components (quantified in the topographic Area Under the Curve or tAUC; Thurlings et al., 2012a,b) and corresponding classification accuracies of a bimodal visual-tactile ERP-BCI enhanced compared to its unimodal counterparts? We hypothesize enhanced bimodal ERP tAUCs both on early and late stages of processing when employing location-congruent bimodal stimuli in a gazeindependent setup, which should result in enhanced classification accuracies.

\section{EFFECTS OF LOCATION-CONGRUENCY ON THE BIMODAL ERP-BCI}

In case we find a benefit of bimodal compared to unimodal stimulus presentation and attending, as hypothesized in the previous section, it is relevant to know whether or not that effect depends (partly) on the spatial relation within the bimodal stimulus pairs. This is important for the designing of bimodal ERP-BCIs, especially since the most straightforward design might employ location incongruent bimodal stimulus pairs as in Thurlings et al. (2012a). In that study, a display presented visual navigation information and included visual stimuli located at the possible navigation directions. Tactile stimuli were presented around the waist, corresponding with navigation directions around us. To make the spatial relation as congruent as possible in that setup, the display was oriented in the horizontal plane, to match the horizontal lay-out of the tactile stimuli (Thurlings et al., 2012b). Therefore the bimodal stimulus pairs were directionalcongruent, but not location-congruent. We showed in Thurlings et al. (2012a) that location-incongruency resulted in negative effects of bimodal (compared to unimodal) stimulus attending on a late stage, while effects on the early stage were positive (see previous section).

Literature on the effects of location-congruency is not unequivocal. According to the spatial rule (Meredith and Stein, 1986), stimuli from different modalities are only integrated when stimuli are spatially co-located (or proximate). Stein et al. (1989) showed for example that the performance of animals that were trained to approach visual stimuli is improved when matched with (unattended) auditory stimuli, but only if the visualauditory stimulus pairs were spatially co-located (or proximate). Frassinetti et al. (2002) replicated these results in humans. However, also when bimodal stimulus-pairs are not locationcongruent, behavior performance has been found to be enhanced (Gondan et al., 2005; Teder-Sälejärvi et al., 2005; Philippi et al., 2008). These studies differ in tasks, but have in common that 
the task does not enforce selective attention to one modality (as in the studies of Meredith and Stein), but rather both modalities need to be attended. Apparently the role of the spatial relation within multisensory information and if and how it affects multisensory integration depends on the specific circumstances (We address the role of selective attention to modality in the next section). Nevertheless, also when bimodal benefits are found for location-incongruent bimodal stimuli, behavioral performance may be further improved by location-congruency (Gondan et al., 2005). Teder-Sälejärvi et al. (2005) did not observe such a behavioral benefit, but did report differences in the ERP for location-congruent and location-incongruent bimodal stimuli after $100 \mathrm{~ms}$. They concluded that there are overlapping and distinct processes involved in processing of location-congruent and incongruent stimuli.

Multisensory studies typically involve a task that requires participants to distinguish targets from nontargets based on physical stimulus characteristics, instead of on (only) spatial differences such as is the case in a BCI-setup (which uses spatial selective attention). Possibly, the role of the spatial relation is larger when the task is only based on spatial discrimination. Therefore it is important to study the role of the spatial relation of bimodal stimuli in ERP-BCIs.

Our second research question is: What is the effect of locationcongruent compared to location-incongruent bimodal stimuli on the ERP tAUCs and corresponding classification accuracies in an ERP-BCI? We hypothesize positive effects for location congruent bimodal stimuli at late stages (e.g., P300 tAUC) of stimulus processing, which should correspond to enhanced classification accuracies.

\section{EFFECTS OF SELECTIVE ATTENTION TO MODALITY ON THE BIMODAL ERP-BCI}

Both exogenous and endogenous attention affect the ERP (Eimer et al., 2001). When participants are presented with bimodal stimuli, but they endogenously attend to either one or both modalities, exogenous attention involved in both cases is the same (as the physical characteristics have not changed). However, the amount of attentional resources allocated endogenously for processing the stimulus information of the two modalities involved differs between these cases (Macaluso, 2010). For example, when participants are precued and (pre)attending to the visual rather than the auditory modality, audio-visual stimuli are processed differently, resulting in enhanced early activity starting around $110 \mathrm{~ms}$ and peaking around $150 \mathrm{~ms}$ (Foxe and Simpson, 2005). Talsma et al. (2007) showed that for the earliest multisensory integration effect (a superadditive effect) of audiovisual stimuli to occur, both modalities need to be attended. Nevertheless, if only a single modality was attended integration still occurred but the process appeared to start later (after $250 \mathrm{~ms}$ after stimulus onset) and was dependent on which modality was attended.

Users of a bimodal ERP-BCI could choose to attend to either one or both modalities, which could affect the resulting ERP and may require modality-specific trained classifiers for optimal performance. In this study, we investigated the tradeoff between possibly affected classification accuracies (when a bimodal classifier trained with attending to both modalities is used also when only one modality is attended to) and the advantage of the flexibility offered to the user to choose the modality to attend to.

Our third research question is: Does, and if so how does, attending to the visual or tactile modality, or both modalities affect ERP components' tAUCs and corresponding classification accuracies in a bimodal ERP-BCI? We hypothesize that when both modalities (as opposed to either one alone) of bimodal (locationcongruent) stimuli are attended, the early stage of the bimodal ERP tAUC is enhanced. Such an enhancement of the ERP tAUC could also result in enhanced classification accuracies.

Following up on the third main research question, a subquestion is: (3a) How do these classification accuracies depend on the degree of overlap in the attended modalities of the datasets during training and classification. That is, would it be possible to switch the attended modality during use, or does the classifier then need to be retrained? We hypothesize that classification accuracies are negatively affected if the applied classifier is trained on data with a different attended modality than the data that are being classified. When attended modalities during training and classifying partly overlap (i.e., visual and bimodal, or tactile and bimodal) higher classification accuracies are expected than when they do not overlap (i.e., visual and tactile).

\section{MATERIALS AND METHODS PARTICIPANTS}

Ten students voluntarily participated in this study. Participants were aged between 22 and 26 years (mean age 23.5 years). All participants were male and had normal or corrected-to-normal vision. None had previously participated in a BCI-experiment and one was left-handed. The participants signed informed consent forms.

\section{TASK}

The task was to select one of two possible control options: left or right. In this study we used the index fingers to present stimulus information, in contrast to locations around the waist (corresponding to navigation directions around us) used in our previous tactile studies. The reason is that we here focus on a gazeindependent and location-congruent setup of bimodal stimuli, and participants should be able to comfortably perceive visual and tactile information from the same location(s). Because the bimodal stimuli should in addition be located at equal distances and angles from fixation, we opted to only employ two bimodal stimuli.

The two control options were presented sequentially in random order, at the left and right index finger through a tactile actuator, an LED, or both. To select an option, participants had to attend to a target stimulus location and modality, and count the number of tactile, visual or visual-tactile activations at that location. At the beginning of each trial the current target (i.e., a combination of finger and modality) was indicated by means of a short activation of the particular target stimulus. Participants were instructed to attend to all targets (and count them internally), and ignore nontargets. Within one trial, each control option (target and nontarget) was activated 10 times. 
Note that although ERP-BCIs typically make use of more than two control options (i.e., more than one nontarget), Brouwer and Van Erp (2010) have shown that the P300 is also elicited in a 2-class tactile BCI and operation is not significantly reduced compared to a 4 - or 6-class BCI.

\section{DESIGN}

The experiment involved six conditions, named after the type of stimuli and attended modality involved. In four conditions targets had to be attended in the modalities that the stimuli of that condition were presented in (no selective attention to modality): Visual, Tactile, Bimodal, Bimodal-Incongr (i.e., short for "Incongruent"). In the Bimodal condition a control option consisted of the simultaneous activation of a visual and tactile actuator at the same finger, while for Bimodal-Incongr a visual and tactile actuator of opposite fingers were matched. In the two other conditions, only one modality had to be attended, while bimodal (location-congruent) stimuli were presented. For attending the visual or tactile modality, the conditions were named: Bimodal-Att- $\mathrm{V}$ and Bimodal-Att- $\mathrm{T}$, respectively. The order of the conditions was counterbalanced over participants.

Each condition consisted of three sets. In each set, each of the two control options was designated as the target three times, i.e., there were six trials. Each trial consisted of 10 consecutive repetitions of the control options in random order, i.e., in each set there were 60 target and 60 nontarget activations. The data of the first two sets (the training sets) were used for the training of a classifier, which was applied to classify the data in the third set (the test set). Online BCI-feedback was given to participants in the test set about which stimulus was classified as the target. The training (but not the test) set was also used for the analysis of participants' ERP components.

\section{MATERIALS}

\section{General}

An actuator pair, consisting of a tactile vibrator and a visual LED, was attached with Velcro to each index finger (22 degrees from a fixation cross). The target and nontarget stimuli consisted of a single pulse with a pulse duration of $187.5 \mathrm{~ms}$. The interval between pulses was $437.5 \mathrm{~ms}$. To indicate the designated target control option at the beginning of a trial and the classified control option for BCI-feedback at the end of a trial, a $2 \mathrm{~s}$ and a $1 \mathrm{~s}$ single pulse were presented, respectively.

\section{Stimuli}

Tactile stimuli: The tactile stimuli were presented through a vibrating element called a tactor. The tactors were custom built and consisted of a plastic case with a contact area of $1 \times 2 \mathrm{~cm}$ containing a $160 \mathrm{~Hz}$ electromotor (TNO, The Netherlands, model JHJ-3, see: Van Erp et al., 2007). To prevent participants from perceiving auditory information from the tactors, they listened to pink noise via speakers during the experiment in all conditions.

Visual stimuli: Visual stimuli were presented through two white LEDs of $5 \mathrm{~mm}, 3.2 \mathrm{~V}$.

Bimodal stimuli: For all bimodal conditions, except for Bimodal-Incongr, bimodal stimuli consisted of the simultaneous activation of the visual and tactile stimulus on the same index finger (location-congruent). For the Bimodal-Incongr condition, the visual stimulus of one index finger and the tactile stimulus of the other index finger were activated simultaneously.

\section{EEG recording equipment}

EEG was recorded from eight linked-mastoids-referenced scalp electrodes $\left(\mathrm{F}_{\mathrm{z}}, \mathrm{C}_{\mathrm{z}}, \mathrm{P}_{\mathrm{z}}, \mathrm{O}_{\mathrm{z}}, \mathrm{P}_{3}, \mathrm{P}_{4}, \mathrm{PO}_{7}, \mathrm{PO}_{8}\right)$ that used a common forehead ground ( $\mathrm{g}$. Tec medical engineering, $\mathrm{GmbH}$ ). The impedance of each electrode was below $5 \mathrm{k} \Omega$, as was confirmed prior to and during the measurements. EEG data were recorded with a hardware filter (bandpass $0.1-60 \mathrm{~Hz}$, notch at $50 \mathrm{~Hz}$ ) and sampled at a frequency of $256 \mathrm{~Hz}$.

\section{DATA ANALYSIS}

\section{EEG preprocessing and selection}

To prepare the recorded EEG for ERP-analysis, we followed similar procedures as taken in Thurlings et al. (2012a,b): selecting (non)target responses, baseline correction, threshold rejection of responses, and computation of a difference ERP. However, the data were not additionally low-pass filtered (the relatively large band was chosen because of potential multisensory effects in the 30-60 Hz band).

Selecting (non)target responses: For ERP-analysis, both target and nontarget responses were used when preceded by a nontarget. Responses preceded by a target were discarded (i.e., there were no (other) targets presented between -625 and $625 \mathrm{~ms}$ relative to (non)target onset) (see also Treder and Blankertz, 2010).

Baseline correction: For the selected targets and nontargets, epochs from all electrodes were extracted from -100 to $625 \mathrm{~ms}$ relative to stimulus onset and baseline corrected relative to the average voltage during the $100 \mathrm{~ms}$ preceding the stimulus onset.

Threshold rejection of responses: We discarded epochs from all electrodes belonging to a certain stimulus, if any epoch contained amplitude differences exceeding $100 \mu \mathrm{V}$, indicating movement artifacts. On average, the previous steps left us with 58.8 target epochs (with a range over participants and conditions from 35 to 70 ) and 54.0 nontarget epochs (with a range over participants and conditions from 32 to 67 ). Subsequently, the selected target and nontarget epochs were averaged per participant, per condition and per electrode.

Difference ERP: We subtracted the averaged clean nontarget epochs from the averaged clean target epochs for each participant, each condition and each electrode. With this step, we removed exogenous (involuntary or automatic) attention effects. Further analyses were performed regarding this difference ERP (or endogenous ERP).

\section{Identifying and quantifying ERP components}

To identify and quantify ERP components triggered by endogenously attended stimuli, we applied the detection method as reported in Thurlings et al. (2012a,b). Only data of the training set was used, to prevent influence of BCI feedback. We identified significant effects of attending stimuli by performing a sampleby-sample $t$-test on the difference ERP (between 0 and $625 \mathrm{~ms}$ relative to (non)target onset) for each electrode and condition and clustered the stable segments (i.e., in this case at least seven consecutive significant samples; see also: Guthrie and Buchwald, 
1991). Clusters were considered robust if they contained segments of at least two electrodes. These robust clusters defined the topographic distribution and the interval of the endogenous ERP components, taking the beginning of the earliest segment and the ending of the latest segment in the clusters as ERP component intervals.

We quantified the endogenous ERP components by using the tAUC-value (topographic Area Under the Curve), as described in Thurlings et al. (2012a,b). The tAUC reflects the magnitude of an ERP component not only by taking the averaged amplitude and duration of the component into account but also by considering the topographic distribution.

\section{Online and offline $\mathrm{BCl}$ performance}

Classification accuracies were calculated both online and offline. Online analysis was performed using BCI2000 (Schalk et al., 2004), which made use of SWLDA (stepwise linear discriminant analysis) on epochs from 0-797 ms after stimulus onset, decimation factor 4 (i.e., $64 \mathrm{~Hz}$ ), and other standard parameters (maximum of 60 features, $p$-values initially included and backward excluded from the model $<0.1$ and $>0.15$ respectively; see Krusienski et al., 2008). The classifier was trained using the training set for each participant and for each condition.

We investigated classification accuracies more detailed offline. Standard classification parameters are based on visual ERP-BCI research. We established potentially more appropriate parameters for bimodal BCIs, and using those parameters assessed the results of the research questions in practical use. To this end, for all conditions we executed a parameter sweep with all combinations of decimation factors (between 4 and 26), and the number of blocks of downsampled windows (between 1 block and the maximum number of blocks approaching a correspondence of $800 \mathrm{~ms}$ ). The parameter-pair resulting in the highest overall classification accuracies (averaged over all six conditions) after 10 repetitions was selected for further analyses. Then for each condition, we calculated accuracies after each repetition and established the number of repetitions which is expected most appropriate in practical use. We considered the number of repetitions the most appropriate, when classification accuracies of $70 \%$ or higher were achieved using a minimal number of repetitions (Kubler et al., 2004; Birbaumer and Cohen, 2007; Pfurtscheller et al., 2010b).

For all conditions, classification accuracies were determined by classifying the test set using a classifier trained on the training set. Additionally, to assess what the costs on BCI performance are of switching attended modality during BCI operation, analysis was done cross-conditionally: that is, for each of the conditions Bimodal, Bimodal-Att-V, and Bimodal-Att- $\mathrm{T}$, the test set was classified using a classifier trained on the test set of each of the other two conditions. From the nine resulting classes of responses, we clustered three categories: (1) trained and tested on data of the same condition ("Equal"); (2) trained and tested on data of two different conditions, but with an overlap in the attended modality (e.g., trained on Bimodal, tested on Bimodal-Att-V; attending of the visual modality is overlapping) ("Overlap"); and (3) trained and tested on data of two different conditions, but without an overlap in the attended modality (e.g., trained on Bimodal-AttV, tested on Bimodal-Att-T ("No Overlap"). Within each category the included classes were averaged per participant.

\section{Statistical analysis}

ERP components' tAUC-values and classification accuracies were statistically analyzed using Statistica 8.0 (StatSoft, Tulsa, USA). To test for normality Shapiro-Wilk tests were applied, and when normality could not be assumed the data were log-transformed. We used separate one-way repeated-measures ANOVAs to examine different subsets of data appropriate to answer each of the three research questions when comparing three conditions, and paired $t$-tests when comparing two conditions. The dependent variables were tAUCs and classification accuracies. For the three main research questions, the independent variables were: (1) Bimodality (three levels: Visual, Tactile, Bimodal); (2) Location-Congruency (two levels: Bimodal, Bimodal-Incongr); and (3) Attending Modality (three levels: Bimodal, Bimodal-AttV, Bimodal-Att-T). For sub-question (3a) the dependent variable was classification accuracies, and the independent variable was Cross-training (three levels: Equal, Overlap, No Overlap). Tukey post-hoc tests were applied when appropriate.

\section{PROCEDURE}

After the participant was verbally instructed and had read and signed the informed consent form, we attached the visual-tactile actuator pairs on his index-fingers using Velcro. The participant was seated in a dimly lit, electromagnetically shielded room and positioned his arms on the desk in front of him. We allowed the participant to become accustomed to the stimuli, by activating them for several minutes. The participant was asked to gaze at the fixation cross in front of him on the table.

During EEG preparation, we repeated the outline of the experiment and instructed the participant to move as little as possible during stimulus presentations. Before each condition, we informed the participant about the oncoming condition. When the participant indicated to be ready to begin, we started the condition. In the test sets, online BCI feedback was given after each trial (i.e., the 10th repetition). Each condition (including two training and one test recording) took approximately $3.8 \mathrm{~min}$ recording time. Conditions followed each other with 1-15 min breaks in between, depending on the participant's preferences.

\section{RESULTS}

First we describe the general observed results (General) considering ERP components and BCI performance for each condition. Subsequently, the effects for each of the three main research questions (regarding the effects of bimodality (The effect of Bimodality), location-congruency (The effect of LocationCongruency), and selective attention to modality (The effect of Selective Attention to Modality)) are reported both with respect to ERP components and classification accuracy. Additionally, the effect of the sub-question of the third main research (addressing the effect of Cross-training) is presented in terms of classification accuracies (Classification accuracies of Cross-training (attended modality cross classifier)). 

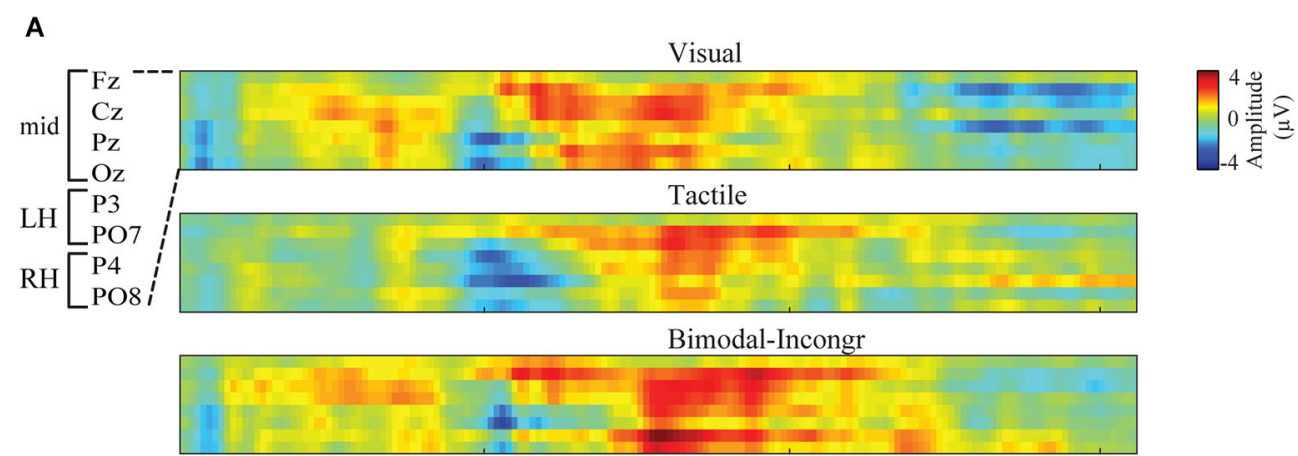

Bimodal

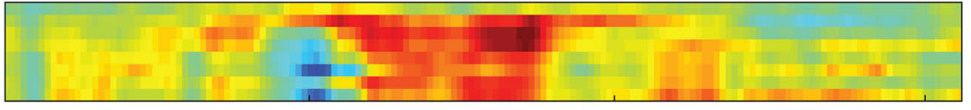

Bimodal-Att-V

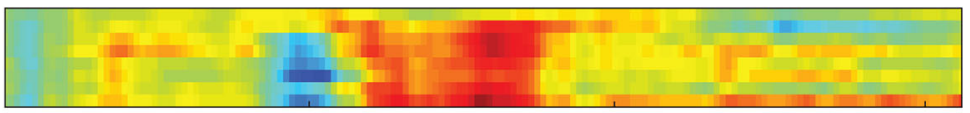

Bimodal-Att-T

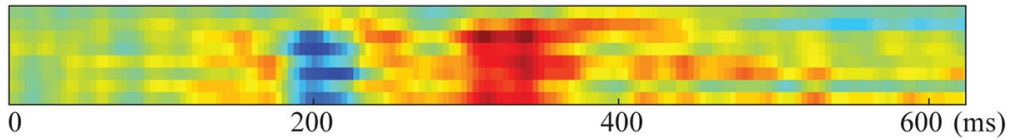

B
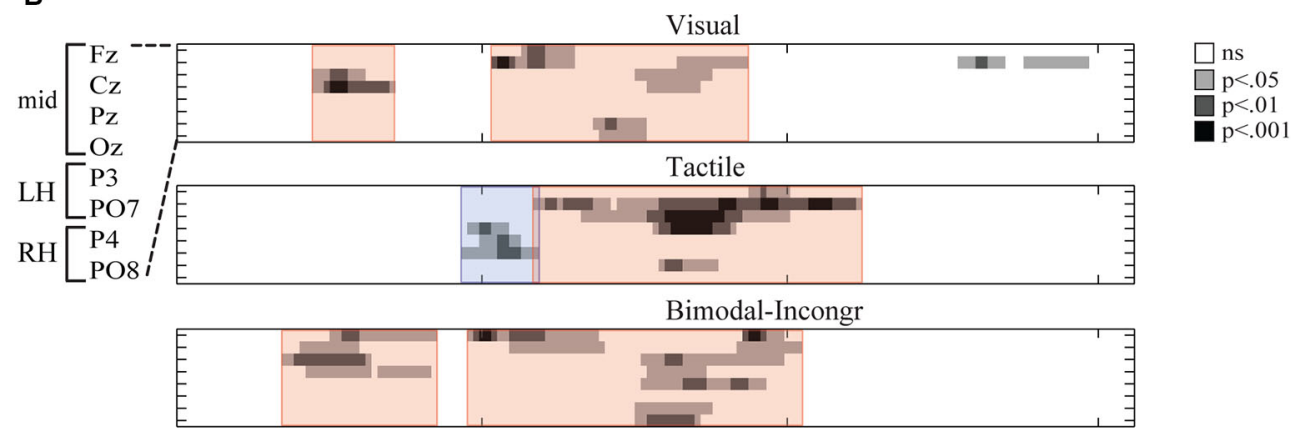

Bimodal

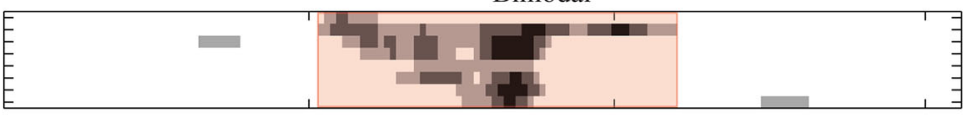

Bimodal-Att-V

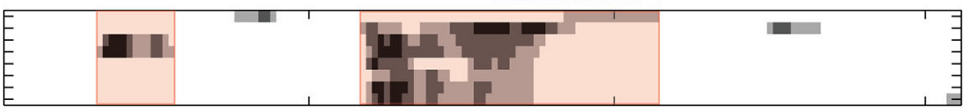

Bimodal-Att-T

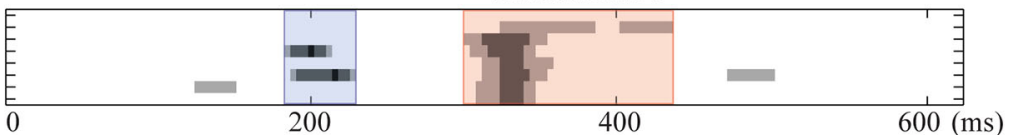

FIGURE 1 | Spatiotemporal representations of the endogenous ERP for each condition, with time (ms) on the $x$-axis and electrodes on the y-axis. Electrodes from top to bottom: $F_{2}, C_{2}, P_{2}, O_{2}, P_{3}, P_{4}, P_{7}, P_{8}$. (A) The Grand Average of the amplitudes of the endogenous ERP $(\mu \mathrm{V})$ for each

\section{GENERAL}

\section{Endogenous ERP components}

Spatiotemporal presentations of the amplitudes of the endogenous ERPs are presented in Figure 1A. For all conditions, condition. (B) The statistical significance of the endogenous ERP ( $p$-values) resulting in stable segments, clustered in ERP components. ERP components are marked by colored overlays in red and blue for positive and negative components, respectively.

endogenous activity was observed during one or two periods within the analyzed interval from 0 until $625 \mathrm{~ms}$ after stimulus onset. In Figure 1B, spatiotemporal plots show the significant stable segments. The red and blue areas in that figure indicate 


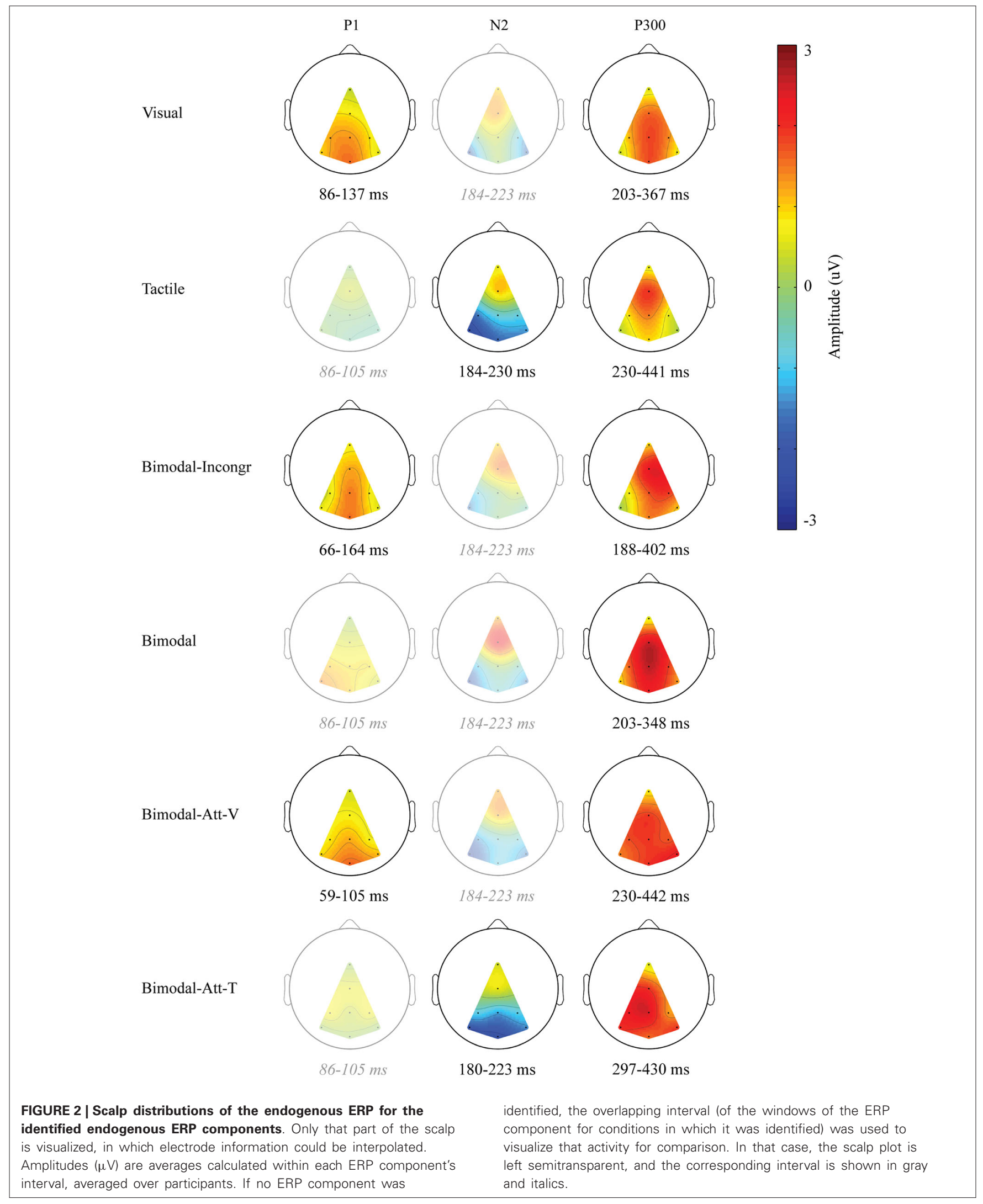


the polarities (positive and negative, respectively) of the clustered segments that were found to be robust and were thus identified as endogenous ERP components.

As apparent from Figure 1B, only one endogenous ERP component was identified in all six conditions: the P300. In Figure 2, the ERP components are visualized by means of scalp plots (averaged amplitudes of the endogenous ERP at all electrodes, within the ERP components' intervals). The P300 amplitudes were largest in the central-parietal area, it appeared to be the strongest in the Bimodal and Bimodal-Att-V conditions, and the weakest for the Visual condition. The windows in which the P300 was detected were: 203-367 ms (Visual), 230-441 ms (Tactile), 188-402 ms (Bimodal-Incongr), 203-348 ms (Bimodal), 230$442 \mathrm{~ms}$ (Bimodal-Att-V), and 297-430 ms (Bimodal-Att-T) after stimulus onset.

Furthermore, early positive activity was detected and identified as $\mathrm{P} 1$ for the Visual, Bimodal-Att-V and Bimodal-Incongr conditions, in the windows 86-137 ms, 59-105 ms, and 66-164 ms respectively, after stimulus onset. For the Tactile and BimodalAtt-T conditions a different early component was detected. Early negative activity was identified as an N2 in the windows 184-230 ms, and 180-223 ms respectively after stimulus onset.

The complete ERPs are visualized for each condition for electrode $\mathrm{P}_{\mathrm{z}}$ in Figure 3 (grouped per research question). The main effect of conditions on the ERP components' tAUC-values are visualized in Figures 5A-C.

\section{BCl performance}

A parameter-sweep was performed for combinations of decimation factors and the length of the epoch used (divided into blocks of downsampled windows). The parameter-pair of decimation factor 5 (i.e., $51.2 \mathrm{~Hz}$ ) and epoch length of $625 \mathrm{~ms}$ resulted in the highest overall classification accuracies of $82.2 \%$ (SD: 11.1 ) over conditions. Thus, these parameters were further used for offline analysis.

Both online and offline classification accuracies are visualized in Figure 4. Overall classification accuracies (averaged over participants) are highest for all bimodal conditions employing location-congruent stimuli (i.e., Bimodal, Bimodal-Att-V, and Bimodal-Att-T). For all conditions offline classification accuracies increase with each repetition, except for the Bimodal-Incongr condition. After six repetitions, the averaged classification accuracies for five out of the six conditions exceeded the threshold of $70 \%$ necessary for effective control. For the Bimodal-Incongr condition this threshold was not reached at all. Therefore the sixth repetition is considered the most appropriate to assess effects of all research questions in a practical setting, and was used for statistical analysis.

\section{THE EFFECT OF BIMODALITY \\ ERP components' tAUCs}

The P300 tAUC (Table 1) was significantly affected by Bimodality $\left(F_{(2,18)}=23.93, p<0.001\right)$. The P300 tAUC was larger for the Bimodal condition compared to both unimodal conditions (both $p<0.001)$, and did not differ significantly between the unimodal conditions (Figure 5A).
The P1 was only identified for the Visual condition (neither for Tactile nor for Bimodal) and the P1's tAUC (Table 1) differed significantly from $0\left(t_{(9)}=6.69, p<0.001\right)$.

The N2 was only identified for the Tactile condition (neither for Visual nor for Bimodal) and the N2's tAUC (Table 1) differed significantly from $0\left(t_{(9)}=6.41, p<0.001\right)$.

\section{Classification accuracies}

The effect of Bimodality on classification accuracies was significant $\left(F_{(2,18)}=7.30, p<0.01\right)$, with higher accuracies for Bimodal compared to Visual $(p<0.05)$ and Tactile $(p<0.01)$ (Figure 5D).

\section{THE EFFECT OF LOCATION-CONGRUENCY ERP components' tAUCs}

An increased P300 tAUC (Table 1) for Bimodal compared to Bimodal-Incongr approached significance $\left(t_{(9)}=2.19, p=0.056\right)$ (Figure 5B).

The P1 was only identified for the Bimodal-Incongr condition (not for Bimodal) and the P1's tAUC (Table 1) differed significantly from $0\left(t_{(9)}=5.91, p<0.001\right)$.

\section{Classification accuracies}

An effect of Location-Congruency on classification accuracies was found, with higher accuracies for Bimodal compared to BimodalIncongr $\left(t_{(9)}=3.88, p<0.01\right)($ Figure $5 \mathrm{E})$.

\section{THE EFFECT OF SELECTIVE ATTENTION TO MODALITY ERP components' tAUCs}

The P300 tAUC was significantly affected by Modality $\left(F_{(2,18)}=7.50, p<0.01\right)$. The P300 was stronger for the Bimodal and Bimodal-Att- $\mathrm{V}$ conditions compared to the Bimodal-Att-T condition ( $p<0.05$ and $p<0.01$, respectively) (Figure 5C).

The P1 was only identified for the Bimodal-Att-V condition (neither for Bimodal-Att-T nor for Bimodal) and the P1's tAUC (Table 1) differed significantly from $0\left(t_{(9)}=8.19\right.$, $p<0.001)$.

The N2 was only identified for the Bimodal-Att-T condition (neither for Bimodal-Att-V nor for Bimodal) and the N2's tAUC (Table 1) differed significantly from $0\left(t_{(9)}=6.20\right.$, $p<0.001)$.

\section{Classification accuracies (attended modality specific classifier)}

For the attended modality specific classifier, the data used for training of the classifier and for the actual classification are recorded under the same attending-modality conditions (using bimodal location-congruent stimuli only).

No effect of Attending Modality on classification accuracies was found (Figure 5F).

\section{Classification accuracies of Cross-training (attended modality cross classifier)}

In this subsection the results of sub-question $3 \mathrm{a}$ are reported. For the attended modality cross classifier, training of the classifier occurred for each of the attending-modality conditions, and the resulting classifier was used to cross-classify the data of each of the attending-modality conditions. 

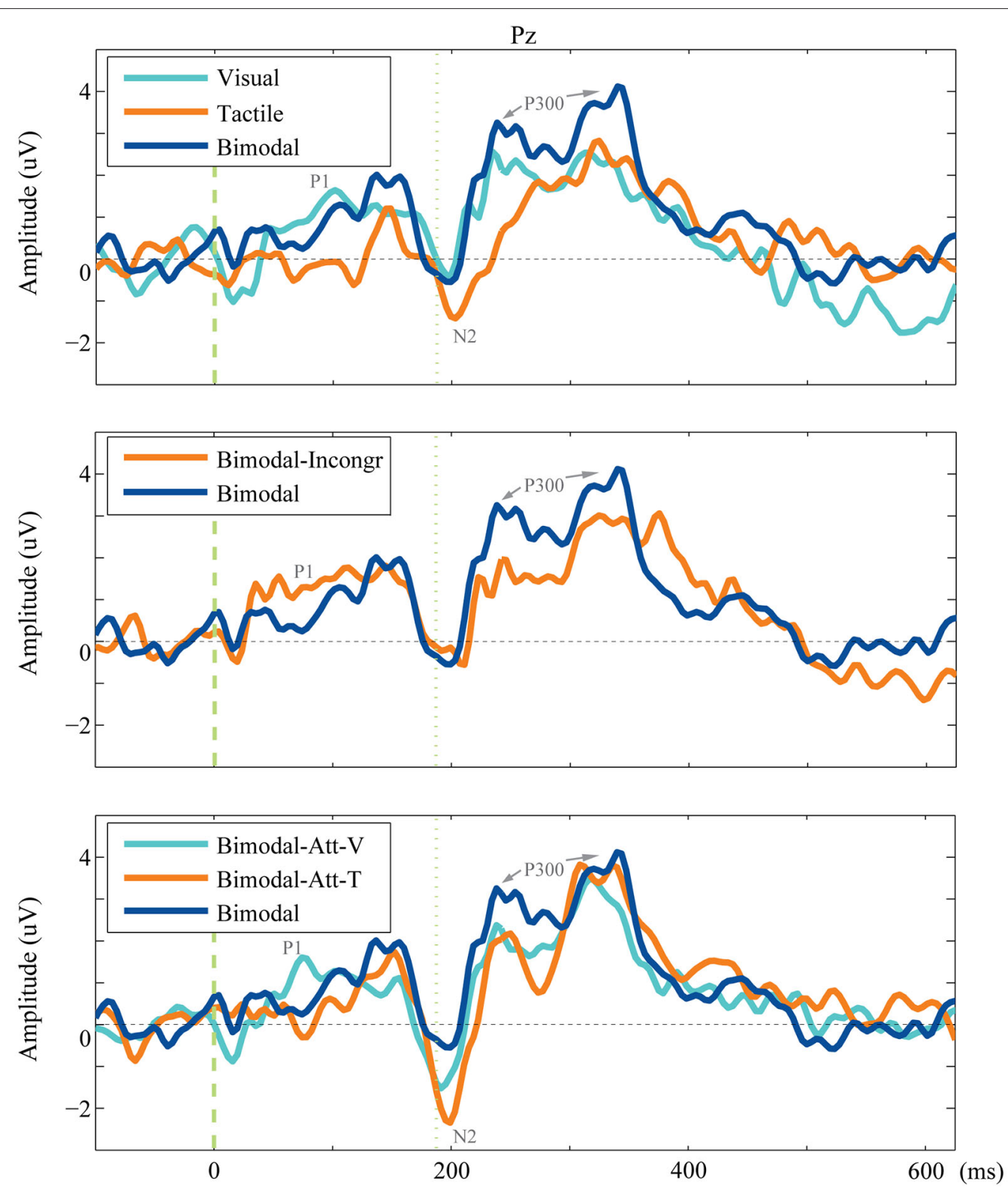

FIGURE 3 | Grand average of the endogenous ERP. The averaged endogenous ERP is visualized for electrode $P_{z}$ for each condition included in the comparison for each research question.

Table 1 | Mean and standard errors averaged over participants of the tAUC-values ( $\mu V^{*} \mathrm{~ms}$ ) of all identified ERP components for each condition.

\begin{tabular}{lcccccc}
\hline ERP Component & Visual & Tactile & Bimodal-Incongr & Bimodal & Bimodal-Att-V & Bimodal-Att-T \\
\hline P1 & $37.6(17.8)$ & & $92.3(49.4)$ & & $36.4(14.1)$ \\
N2 & $168.9(63.5)$ & $237.0(73.4)$ & $351.3(140.9)$ & $526.7(223.3)$ & $534.7(125.4)$ & $265.2(168.0)$ \\
P300 & & &
\end{tabular}

Table 2 shows the results of the cross-condition classification analyses. In Figure 5G the effect of Cross-training on the clustered categories is visualized. Cross-training affected classification accuracies $\left(F_{(2,18)}=4.86, p<0.05\right)$, with higher accuracies for Equal compared to No Overlap $(p<0.05)$.

\section{DISCUSSION}

\section{THE EFFECT OF BIMODALITY}

The first and main research question addressed in this study was: Are ERP components' tAUCs and corresponding classification accuracies of a bimodal visual-tactile ERP-BCI enhanced 

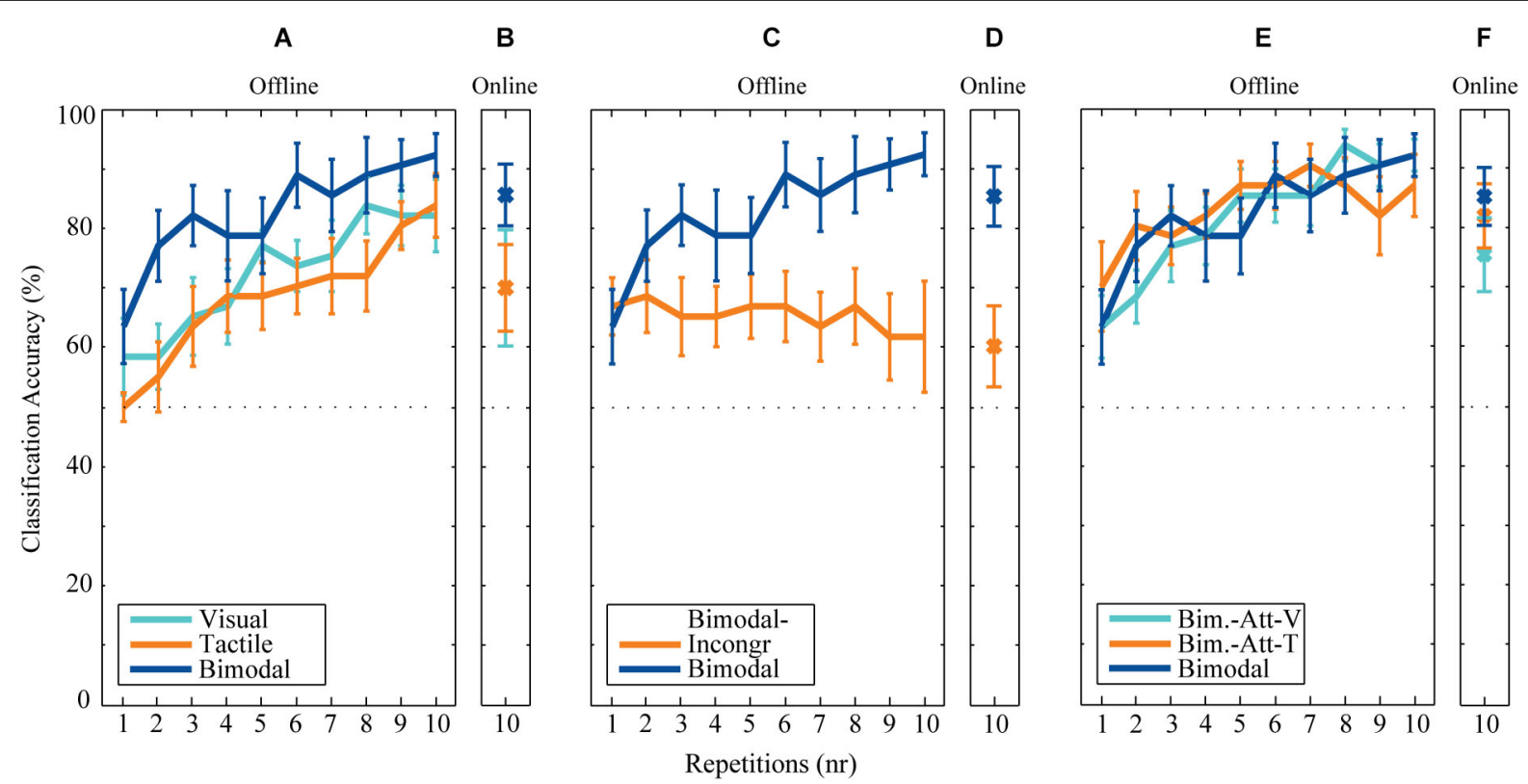

FIGURE 4 | Offline and online classification accuracies. (A) Offline and (B) online classification accuracies after each repetition for the conditions

repetition for the conditions involved in the analysis of the second research question (the effect of Location Congruency). (E) Offline and (F) online involved in the analysis of the first research question (the effect of classification accuracies after each repetition for the conditions involved in the Bimodality). (C) Offline and (D) online classification accuracies after each analysis of the third research question (the effect of Attending Modality).
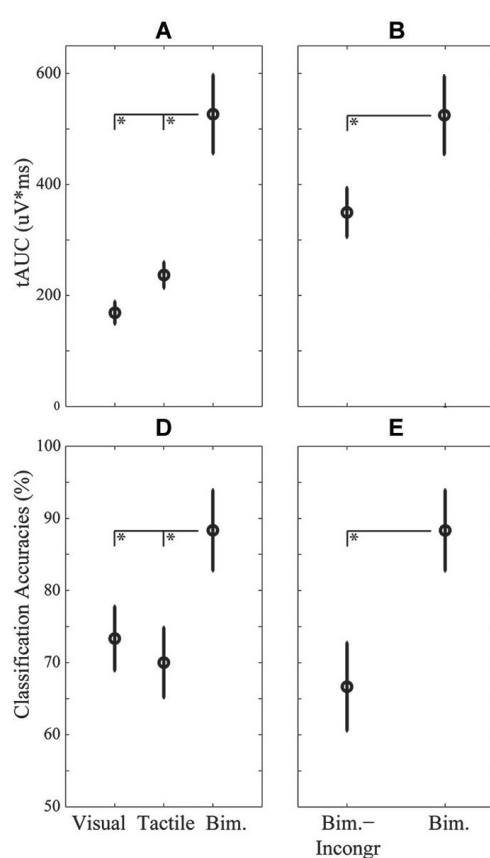

B
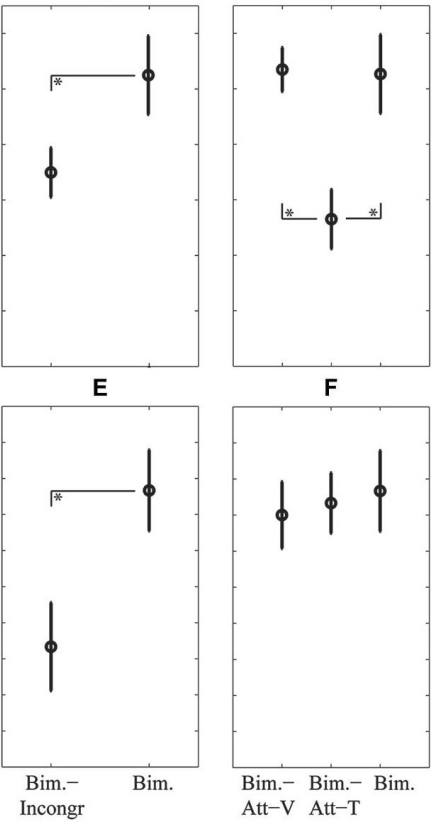

C
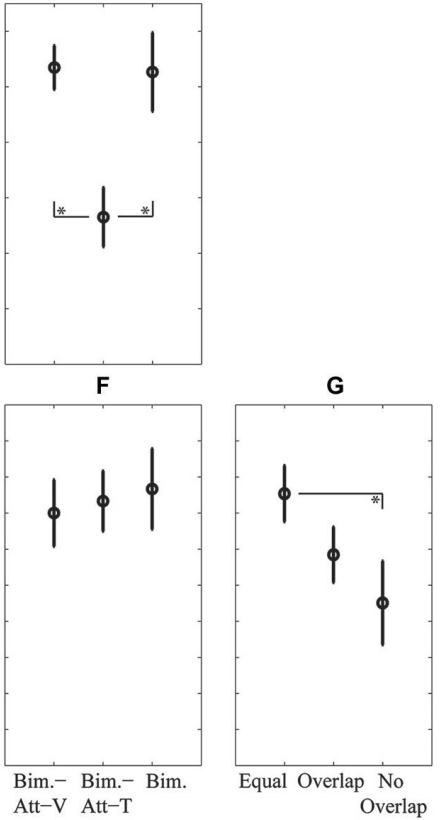

FIGURE 5 | Mean and standard errors over participants of the P300 (A-C) and classification accuracies (D-G), for each research question: the effect of modality $(A, D)$, the effect of location-congruency $(B, E)$, the effect of selective attention to modality $(C, F)$, and the effect of cross-training (G). Condition pairs that significantly differed from each other are indicated by an asterisk $\left({ }^{*}\right)$ symbol. 
Table 2 | Classification accuracies (averages and standard deviations) for each class of cross-conditional classification.

\begin{tabular}{lccc}
\hline Tested on & $\begin{array}{c}\text { Trained on } \\
\text { Bimodal }\end{array}$ & Bimodal-Att-V & Bimodal-Att-T \\
\hline Bimodal & $83.33(17.66)^{*}$ & $76.67(21.08)^{\wedge}$ & $80.00(18.92)^{\wedge}$ \\
Bimodal-Att-V & $81.67(12.30)^{\wedge}$ & $85.00(14.59)^{*}$ & $73.33(14.05)^{\#}$ \\
Bimodal-Att-T & $76.67(17.92)^{\wedge}$ & $71.67(26.12)^{\#}$ & $86.67(13.15)^{*}$
\end{tabular}

Symbols $* \wedge \#$ indicate which classes are categorized together, with * for Equal, ^ for Overlap, and ${ }^{\#}$ for No Overlap.

compared to its unimodal counterparts? As we hypothesized, we found an enhanced late effect on the ERP (P300 tAUC) and corresponding enhanced classification accuracies for the locationcongruent bimodal compared to the unimodal conditions, using a gaze-independent setup. In our previous bimodal work (Thurlings et al., 2012a), we did not find an enhanced bimodal P300. Instead, the bimodal P300 was even decreased compared to the visual P300. We hypothesized that effect to be a result of location-incongruent bimodal stimuli, as the P300 is affected by spatial attention (Kramer and Strayer, 1988). In this study, we showed that attending to (location-congruent) bimodal (compared to unimodal) stimuli does indeed result in an increasement of the P300 tAUC. The different findings in these two studies hint that location-congruency may indeed affect the processing of bimodal stimuli, which we will further discuss in the next section (The effect of Location-Congruency).

In contrast to our expectations, we did not find positive effects of attending to bimodal stimuli on the early stage of processing. In fact, we did not detect an early bimodal ERP component at all for location-congruent stimuli when both modalities were attended. However we did observe early ERP components for both unimodal conditions: a visual P1 and a tactile N2. Because the unimodal conditions resulted in early ERP components with opposite polarities, the lack of a bimodal early ERP component in this study may be explained by counterbalanced activity. In Thurlings et al. (2012a) we did find a bimodal early ERP component (N1), which was not detected in either of the unimodal conditions. The early ERPs of those unimodal conditions, however, appeared much more alike and already showed a slight negative drift. Also in Talsma and Woldorff (2005)—in which positive effects of audiovisual stimulus attending on early and late stages of processing are reported- the unimodal early ERPs were quite alike. The same is the case in other bimodal studies (e.g., Gondan et al., 2005; Teder-Sälejärvi et al., 2005; Philippi et al., 2008). In multisensory literature there is an on-going debate about whether or not superadded activity is elicited when multisensory integration takes place, and how integration effects can be measured (Barth et al., 1995; Gondan and Röder, 2006; Boll and Berti, 2009; Senkowski et al., 2011). Perhaps ERP summation is the driving factor behind enhanced effects of bimodal compared to unimodal ERPs in our study.

If unimodal ERP components need to be alike to elicit bimodal effects usable in BCI, it is relevant to understand why in the current study this was not the case. For the tactile condition the early ERP component (N1) resembles the tactile N2 described in Thurlings et al. (2012a), but occurred slightly
( $25 \mathrm{~ms}$ ) earlier in this study. The P1 from the visual condition also occurs $\sim 25 \mathrm{~ms}$ earlier compared to the visual N2 in Thurlings et al. (2012a), but has a reversed polarity. Possibly, these visual early components do have the same generator: The polarity of the P1 can be reversed if the concerning electrode is measured in reference to for example the nose instead of linked-mastoids (Chiappa and Chiappa, 1997). Indeed in this study linked-mastoid references were used (with which a visual P1 is expected: Mangun, 1995) while in Thurlings et al. (2012a) a nose-reference was used. Not every ERP component has to be affected by such a difference: depending on the generator of a certain ERP component and the recorded electrode(s) this can affect polarity.

Attending to bimodal compared to unimodal stimuli may have increased exogenous as well as endogenous attention. Therefore the cause of the bimodality effect here could theoretically be either bottom-up, or top-down driven, or by an interaction between the two. In this study Bimodality affected the ERP positively at the late stage. Since Hopfinger and West (2006) found the P300 to be unaffected by increased exogenous attention, we think that top-down controlled endogenous attention caused the Bimodality effect. While this study does not map out the exact mechanism behind the effect, it is clear that BCI performance was much higher when congruent bimodal stimuli were used compared to unimodal stimuli. We therewith provide a way to improve performance of a gaze-independent ERP-BCI.

\section{THE EFFECT OF LOCATION-CONGRUENCY}

The second research question concerned the effect of locationcongruent compared to location-incongruent bimodal stimuli on the ERP components' tAUC and corresponding classification accuracies in an ERP-BCI. As we hypothesized, we found an indication that location-congruency positively affects the late ERP component tAUC in response to bimodal stimuli $(p=0.056)$, and this trend corresponded to increased classification accuracies.

Although we only expected location-congruency to influence the late stage of the ERP, we also found a difference at the early stage: A P1 was observed for the Bimodal-Incongr condition, whereas we did not detect early ERP components for the Bimodal condition at all. This $\mathrm{P} 1$ resembles the $\mathrm{P} 1$ from the conditions in which the visual modality was relevant (Visual and BimodalAtt-V). Therefore the occurrence of the P1 in the BimodalIncongr condition could be due to (stronger) attending to the visual modality. Although for that condition, participants were instructed to attend both modalities equally, the task may have been too difficult as the locations of the visual and tactile parts of the bimodal incongruent stimuli were rather far apart, and even in opposite hemifields. This could have caused participants to attend more to one of the modalities, in this case, the visual modality. The P1 seems even stronger in the Bimodal-Incongr compared to the Visual condition and compared to the BimodalAtt- $\mathrm{V}$ condition, suggesting that in the Bimodal-Incongr condition participants tried to focus even more on the visual part of the stimulus to not have themselves distracted by the tactile stimulus.

BCI performance was clearly affected by location-congruency. Therefore bimodal BCIs (based on spatial attention) should be 
based on location-congruent bimodal stimuli for optimal performance. The performance drop caused by location-incongruent bimodal stimuli is expected to depend on the degree of incongruency.

\section{THE EFFECT OF SELECTIVE ATTENTION TO MODALITY}

The third research question was does, and if so how does, attending to the visual or tactile modality, or both modalities affect ERP components' tAUCs and corresponding classification accuracies in a bimodal ERP-BCI? We hypothesized a positive effect on the late ERP component tAUC when both modalities of bimodal (location-congruent) stimuli were attended rather than just one. Indeed attending to both modalities resulted in a stronger P300 compared to attending to the tactile modality alone, but it was equally strong as attending to the visual modality alone. Possibly, and in line with our interpretation of effects on the $\mathrm{P} 1$ as discussed in the previous section (processing of), the visual stimulus was dominant over (processing of) the tactile stimulus.

Selectively attending modality also had an effect on the early ERP components' tAUCs. When the visual modality was attended in a bimodal BCI, a P1 was detected, similar as in the visual BCI. Likewise, when the tactile modality was attended in a bimodal BCI, an N2 was detected, similar as for the tactile BCI. Thus, these early ERP effects appear unrelated to multisensory interaction and solely explainable by unisensory (bottom-up) effects of stimulus processing at attended locations and within attended modalities.

\section{The effect of Cross-training}

BCI performance in terms of classification accuracies was equally good for the three bimodal attention conditions. This means that users can choose a preferred modality to attend to for operating a bimodal BCI when training and classifying occurs using the same attended modalities (attended modality specific classifier). We additionally assessed the effect of switching the attended modality during BCI operation by cross-classifying each one of the three bimodal attention conditions (sub-question $3 \mathrm{a})$. The results indicate that when the attended modalities are different during bimodal BCI operation and training of the bimodal classifier (attended modality cross classifier), this causes a drop in BCI performance. The size of this drop depends on the degree of overlap in the attended modalities. However, even if there is no overlap, operation of the bimodal BCI is still feasible and performance is similar to that of unimodal BCIs. That means that bimodal BCIs offer the option to be used flexibly, i.e., users can switch the modality to attend to during operation.

\section{CONCLUSION}

Multisensory effects can be used to enhance BCI performance (as reflected by classification accuracies) by employing bimodal stimuli. In this study we investigated bimodal effects in gaze-independent ERP-BCIs, using visual-tactile stimuli. The P300 tAUC and corresponding classification accuracies were enhanced when participants were attending to (locationcongruent) bimodal vs. unimodal stimuli. Unexpectedly, we did not observe early bimodal effects for the specific condition where stimuli were location-congruent and both modalities were attended. This is possibly due to reversed polarities of early unimodal ERP components. We suggest that bimodal BCI performance may further be improved when the early unimodal ERP components are more similar, which may be achieved with different locations of the EEG reference electrode.

Furthermore, bimodal classification accuracies were improved when bimodal stimuli were location-congruent. Thus bimodal BCIs should be designed location-congruent for optimal performance.

Additionally, BCI performance was invariant for the specific modality attended, although the underlying ERP components' tAUCs were affected. When the bimodal classifier was not trained for the specific modality attended, the drop in BCI performance depends on the degree of overlap in attended modalities between training and classifying, but was still at least as good as for the unimodal ERP-BCIs. Thus bimodal BCIs may increase BCI performance and offer more flexibility in use. This implies that for the practical use of BCIs, people who are either restricted physically or by the context of use (e.g., sensory overload of the visual channel while driving) to attend to a certain modality may benefit from using bimodal BCIs.

\section{ACKNOWLEDGMENTS}

This research has been supported by the GATE project, funded by the Netherlands Organization for Scientific Research (NWO) and the Netherlands ICT Research and Innovation Authority (ICT Regie). The authors gratefully acknowledge the support of the BrainGain Smart Mix Programme of the Netherlands, the Ministry of Economic Affairs and the Netherlands Ministry of Education, Culture and Science.

We also like to thank the students of the elective course "Multimodal Perception and Interaction" at the Utrecht University, and Antoon Wennemers.

\section{REFERENCES}

Acqualagna, L., and Blankertz, B. (2013). Gaze-independent BCI-spelling using rapid serial visual presentation (RSVP). Clin. Neurophysiol. 124, 901-908. doi: 10.1016/j.clinph.2012.12.050

Barth, D. S., Goldberg, N., Brett, B., and Di, S. (1995). The spatiotemporal organization of auditory, visual and auditory-visual evoked potentials in rat cortex. Brain Res. 678, 177-190. doi: 10.1016/0006-8993(95)00182-p

Belitski, A., Farquhar, J., and Desain, P. (2011). P300 audio-visual speller. J. Neural Eng. 8:025022. doi: 10.1088/1741-2560/8/2/025022

Bell, C. J., Shenoy, P., Chalodhorn, R., and Rao, R. P. N. (2008). Control of a humanoid robot by a noninvasive brain-computer interface in humans. J. Neural Eng. 5, 214-220. doi: 10.1088/1741-2560/5/2/012

Birbaumer, N., and Cohen, L. G. (2007). Brain-computer interfaces: communication and restoration of movement in paralysis. J. Physiol. 579, 621-636. doi: 10. 1113/jphysiol.2006.125633

Boll, S., and Berti, S. (2009). Distraction of task-relevant information processing by irrelevant changes in auditory, visual and bimodal stimulus features: a behavioral and event-related potential study. Psychophysiology 46, 645-654. doi: 10.1111/j.1469-8986.2009.00803.x

Brouwer, A.-M., and Van Erp, J. B. F. (2010). A tactile P300 brain-computer interface. Front. Neurosci. 4:19. doi: 10.3389/fnins.2010.00019

Brouwer, A.-M., Van Erp, J. B. F., Aloise, F., and Cincotti, F. (2010). Tactile, visual and bimodal P300s: could bimodal P300s boost BCI performance? SRX Neurosci. 2010. doi: 10.3814/2010/967027 
Brunner, P., Joshi, S., Briskin, S., Wolpaw, J. R., Bischof, H., and Schalk, G. (2010). Does the 'P300' speller depend on eye gaze? J. Neural Eng. 7:056013. doi: 10. 1088/1741-2560/7/5/056013

Chiappa, K. H., and Chiappa, K. H. (1997). Pattern-shift visual evoked potentials: methodology. Evoked Potentials Clin. Med. 2, 37-109.

Driver, J., and Noesselt, T. (2008). Multisensory interplay reveals crossmodal influences on 'sensory-specific' brain regions, neural responses and judgments. Neuron 57, 11-23. doi: 10.1016/j.neuron.2007.12.013

Eimer, M., Cockburn, D., Smedley, B., and Driver, J. (2001). Cross-modal links in endogenous spatial attention are mediated by common external locations: evidence from event-related brain potentials. Exp. Brain Res. 139, 398-411. doi: $10.1007 /$ s002210100773

Ernst, M. O., and Bülthoff, H. H. (2004). Merging the senses into a robust percept. Trends Cogn. Sci. 8, 162-169. doi: 10.1016/j.tics.2004.02.002

Farwell, L. A., and Donchin, E. (1988). Talking off the top of your headtoward a mental prosthesis utilizing event-related brain potentials. Electroencephalogr. Clin. Neurophysiol. 70, 510-523. doi: 10.1016/0013-4694(88) 90149-6

Foxe, J. J., and Simpson, G. V. (2005). Biasing the brain's attentional set: II. Effects of selective intersensory attentional deployments on subsequent sensory processing. Exp. Brain Res. 166, 393-401. doi: 10.1007/s00221-0052379-6

Frassinetti, F., Bolognini, N., and Ladavas, E. (2002). Enhancement of visual perception by crossmodal visuo-auditory interaction. Exp. Brain Res. 147, 332 343. doi: 10.1007/s00221-002-1262-y

Giray, M., and Ulrich, R. (1993). Motor coactivation revealed by response force in divided and focused attention. J. Exp. Psychol. Hum. Percept. Perform. 19, 1278 1291. doi: 10.1037/0096-1523.19.6.1278

Gondan, M., Niederhaus, B., Rösler, F., and Röder, B. (2005). Multisensory processing in the redundant-target effect: a behavioral and event-related potential study. Percept. Psychophys. 67, 713-726. doi: 10.3758/bf03193527

Gondan, M., and Röder, B. (2006). A new method for detecting interactions between the senses in event-related potentials. Brain Res. 1073-1074, 389-397. doi: 10.1016/j.brainres.2005.12.050

Guthrie, D., and Buchwald, J. S. (1991). Significance testing of difference potentials. Psychophysiology 28, 240-244. doi: 10.1111/j.1469-8986.1991. tb00417.x

Höhne, J., Schreuder, M., Blankertz, B., and Tangermann, M. (2011). A novel 9-class auditory erp paradigm driving a predictive text entry system. Front. Neurosci. 5:99. doi: 10.3389/fnins.2011.00099

Hopfinger, J. B., and West, V. M. (2006). Interactions between endogenous and exogenous attention on cortical visual processing. Neuroimage 31, 774-789. doi: 10.1016/j.neuroimage.2005.12.049

Kramer, A. F., and Strayer, D. L. (1988). Assessing the development of automatic processing: an application of dual-task and event-related brain potential methodologies. Biol. Psychol. 26, 231-267. doi: 10.1016/0301-0511(88) 90022-1

Krusienski, D. J., Sellers, E. W., McFarland, D. J., Vaughan, T. M., and Wolpaw, J. R. (2008). Toward enhanced P300 speller performance. J. Neurosci. Methods 167, 15-21. doi: 10.1016/j.jneumeth.2007.07.017

Kubler, A., Neumann, N., Wilhelm, B., Hinterberger, T., and Birbaumer, N. (2004). Brain-computer predictability of brain-computer communication. J. Psychophysiol. 18, 121-129. doi: 10.1027/0269-8803.18.23.121

Macaluso, E. (2010). Orienting of spatial attention and the interplay between the senses. Cortex 46, 282-297. doi: 10.1016/j.cortex.2009.05.010

Mangun, G. R. (1995). Neural mechanisms of visual selective attention. Psychophysiology 32, 4-18. doi: 10.1111/j.1469-8986.1995.tb03400.x

Meredith, M. A., and Stein, B. E. (1986). Spatial factors determine the activity of multisensory neurons in cat superior colliculus. Brain Res. 365, 350-354. doi: 10. 1016/0006-8993(86)91648-3

Molholm, S., Ritter, W., Murray, M. M., Javitt, D. C., Schroeder, C. E., and Foxe, J. J. (2002). Multisensory auditory-visual interactions during early sensory processing in humans: a high-density electrical mapping study. Brain Res. Cogn. Brain Res. 14, 115-128. doi: 10.1016/s0926-6410(02)00066-6

Orhan, U., Ii, K. E. H., Erdogmus, D., Roark, B., Oken, B., and Fried-oken, M. (2012). RSVP keyboard: an EEG based typing interface. Acoustics, Speech and Signal Processing (ICASSP), 2012 IEEE International Conference on (Kyoto), 645-648.
Pfurtscheller, G., Allison, B. Z., Brunner, C., Bauernfeind, G., Solis-Escalante, T., Scherer, R., et al. (2010a). The hybrid BCI. Front. Neurosci. 4:30. doi: 10. 3389/fnpro.2010.00003

Pfurtscheller, G., Solis-Escalante, T., Ortner, R., Linortner, P., and Muller-Putz, G. R. (2010b). Self-paced operation of an ssvep-based orthosis with and without an imagery-based "brain switch:" a feasibility study towards a hybrid BCI. IEEE Trans. Neural Syst. Rehabil. Eng. 18, 409-414. doi: 10.1109/tnsre.2010.20 40837

Philippi, T. G., Van Erp, J. B. F., and Werkhoven, P. J. (2008). Multisensory temporal numerosity judgment. Brain Res. 1242, 116-125. doi: 10.1016/j.brainres.2008. 05.056

Sambo, C. F., and Forster, B. (2009). An ERP investigation on visuotactile interactions in peripersonal and extrapersonal space: evidence for the spatial rule. $J$. Cogn. Neurosci. 21, 1550-1559. doi: 10.1162/jocn.2009.21109

Schalk, G., McFarland, D. J., Hinterberger, T., Birbaumer, N., and Wolpaw, J. R. (2004). BCI2000: a general-purpose, brain-computer interface (BCI) system. IEEE Trans. Biomed. Eng. 51, 1034-1043. doi: 10.1109/tbme.2004. 827072

Schreuder, M., Blankertz, B., and Tangermann, M. (2010). A new auditory multiclass brain-computer interface paradigm: spatial hearing as an informative cue. PLoS One 5:e9813. doi: 10.1371/journal.pone.0009813

Schreuder, M., Rost, T., and Tangermann, M. (2011). Listen, you are writing! speeding up online spelling with a dynamic auditory BCI. Front. Neurosci. 5:112. doi: $10.3389 /$ fnins. 2011.00112

Schröger, E., and Widmann, A. (1998). Speeded responses to audiovisual signal changes result from bimodal integration. Psychophysiology 35, 755-759. doi: 10. 1111/1469-8986.3560755

Senkowski, D., Saint-Amour, D., Hofle, M., and Foxe, J. J. (2011). Multisensory interactions in early evoked brain activity follow the principle of inverse effectiveness. Neuroimage 56, 2200-2208. doi: 10.1016/j.neuroimage.2011. 03.075

Stein, B. E., Meredith, M. A., Huneycutt, W. S., and McDade, L. (1989). Behavioral indices of multisensory integration: orientation to visual cues is affected by auditory stimuli. J. Cogn. Neurosci. 1, 12-24. doi: 10.1162/jocn.1989. 1.1 .12

Stein, B. E., and Stanford, T. R. (2008). Multisensory integration: current issues from the perspective of the single neuron. Nat. Rev. Neurosci. 9, 255-266. doi: 10. $1038 /$ nrn2377

Talsma, D., Doty, T. J., and Woldorff, M. G. (2007). Selective attention and audiovisual integration: is attending to both modalities a prerequisite for early integration? Cereb. Cortex 17, 679-690. doi: 10.1093/cercor/ bhk016

Talsma, D., and Woldorff, M. G. (2005). Selective attention and multisensory integration: multiple phases of effects on the evoked brain activity. J. Cogn. Neurosci. 17, 1098-1114. doi: 10.1162/0898929054475172

Teder-Sälejärvi, W. A., Di Russo, F., McDonald, J. J., and Hillyard, S. A. (2005). Effects of spatial congruity on audio-visual multimodal integration. J. Cogn Neurosci. 17, 1396-1409. doi: 10.1162/0898929054985383

Thurlings, M. E., Brouwer, A. M., Van Erp, J. B. F., Blankertz, B., and Werkhoven, P. J. (2012a). Does bimodal stimulus presentation increase ERP components usable in BCIs? J. Neural Eng. 9:045005. doi: 10.1088/1741-2560/9/4/ 045005

Thurlings, M. E., Van Erp, J. B. F., Brouwer, A.-M., Blankertz, B., and Werkhoven, P. J. (2012b). Control-display mapping in brain-computer interfaces. Ergonomics 55, 564-580. doi: 10.1080/00140139.2012.661085

Thurlings, M. E., Van Erp, J. B. F., Brouwer, A.-M., and Werkhoven, P. J. (2010). "EEG-based navigation from a human factors perspective," in Brain-Comput. Interfaces, eds D. S. Tan and A. Nijholt (London: Springer), 71-86.

Thurlings, M. E., Van Erp, J. B. F., Brouwer, A.-M., and Werkhoven, P. (2013). Controlling a tactile ERP-BCI in a multimodal dual-task. IEEE Trans. Comput. Intell. AI in Games 5, 129-140. doi: 10.1109/tciaig.2013.2239294

Treder, M. S., and Blankertz, B. (2010). (C)overt attention and visual speller design in an ERP-based brain-computer interface. Behav. Brain Funct. 6:28. doi: 10 1186/1744-9081-6-28

Van Erp, J. B. F. (2005). Presenting directions with a vibrotactile torso display. Ergonomics 48, 302-313. doi: 10.1080/0014013042000327670

Van Erp, J. B. F., Eriksson, L., Levin, B., Carlander, O., Veltman, J. A., and Vos, W. K. (2007). Tactile cueing effects on performance in simulated aerial combat with 
high acceleration. Aviat. Space Environ. Med. 78, 1128-1134. doi: 10.3357/asem. 2133.2007

Van Erp, J. B. F., and Van Veen, H. A. H. C. (2004). Vibrotactile in-vehicle navigation system. Transp. Res. Part F Traffic Psychol. Behav. 7, 247-256. doi: 10.1016/j.trf. 2004.09.003

Van Erp, J. B. F., Veltman, J. A., and Grootjen, M. (2010). "Brain-based indices for user system symbiosis," in Brain Computer Interfaces, Human-Computer Interaction Series, eds D. S. Tan and A. Nijholt (London: Spinger-Verlag), 201-219.

Woldorff, M. G., and Hillyard, S. A. (1991). Modulation of early auditory processing during selective listening to rapidly presented tones. Electroencephalogr. Clin. Neurophysiol. 79, 170-191. doi: 10.1016/0013-4694(91) 90136-r

Yin, E., Zhou, Z., Jiang, J., Chen, F., Liu, Y., and Hu, D. (2013). A novel hybrid BCI speller based on the incorporation of SSVEP into the P300 paradigm. J. Neural Eng. 10:026012. doi: 10.1088/1741-2560/10/2/026012
Conflict of Interest Statement: The authors declare that the research was conducted in the absence of any commercial or financial relationships that could be construed as a potential conflict of interest.

Received: 03 February 2014; accepted: 23 July 2014; published online: 08 September 2014.

Citation: Thurlings ME, Brouwer A-M, Van Erp JBF and Werkhoven P (2014) Gazeindependent ERP-BCIs: augmenting performance through location-congruent bimodal stimuli. Front. Syst. Neurosci. 8:143. doi: 10.3389/fnsys.2014.00143

This article was submitted to the journal Frontiers in Systems Neuroscience.

Copyright $\odot 2014$ Thurlings, Brouwer, Van Erp and Werkhoven. This is an open-access article distributed under the terms of the Creative Commons Attribution License (CC BY). The use, distribution or reproduction in other forums is permitted, provided the original author(s) or licensor are credited and that the original publication in this journal is cited, in accordance with accepted academic practice. No use, distribution or reproduction is permitted which does not comply with these terms. 